\title{
Solution of some stochastic differential equation
}

\author{
Dr. Sana Hussein
}

Dept .of Mathematics, Faculty of Science, Sudan University of Science and technology. ,King Feisal University, Saudi Arabia

Abstract: In this paper we study the method of solution of some stochastic differential equations of first order by using the ito integral and ito formula

$$
\text { في هذه الورقة تمتة در اسة طرق طلول بعض المعادلات التفاضلية التصادفية ذات الرتبة الاولي باستخدام تكامل وصيغة اتو }
$$

Key words: stochastic differential equations, ito integral ito formula

\section{I.}

\section{Introduction:}

Consider the simple population growth model

$\frac{d N}{d t}=a(t) N(t), N(0)=N_{0}$ (constant) (1)

Where $N(t)$, is the size of population at time $t$ and $a(t)$ is the rate of growth at time $t . \quad a(t)$ Is not completely known

$a(t)=r(t)+$ noise term where We don't know the exact behavior of the noise term, the function $r(t)$ is assumed to be non random

How do we solve (1) in this case??

In this paper we discuss the method for solving similar of the above example.

(2)The basic concept of stochastic differential equations

\section{1-probability space:}

The triple $(\Omega, \mathcal{F}, P)$ is called probability space.

\section{2-stochastic process:}

A stochastic process is a collection of random variables

$\left\{X_{t}\right\}_{t \in \in T}, X_{t}: \Omega \rightarrow R^{n}$ Defined on $(\Omega, \mathcal{F}, P)$.

\section{3-Brownian motion:}

A Brownian motion is a random variable satisfies the following:

$1-B(0)=0$

$2-B(t)$ is continuous functions of $\mathrm{t}$

3- $B(t)$ Has independent normally distributed increments

Consider the example

\section{$\underline{\text { 2-Ito integral: }}$}

$$
\frac{d N}{d t}=(r(t)+\text { noise term }) N(t)
$$

Or

$\frac{d X}{d t}=b\left(t, X_{t}\right)+\sigma\left(t, X_{t}\right)$. noise term

Where $w_{t}$ the white noise $X_{t}$ is the stochastic process

By integrating example (2) from $k$ into $k+1$ we get

$$
\begin{gathered}
\int_{k}^{k+1} \frac{d X}{d t}=\int_{k}^{k+1} b\left(t, X_{t}\right) d t+\sigma\left(t, X_{t}\right) .(\text { noise }) d t \\
X_{k+1}-X_{k}=b\left(t_{k}, X_{k}\right) \Delta t_{k}+\sigma\left(t_{k}, X_{k}\right) w_{k} \Delta t_{k} \\
\ni \Delta t_{k}=t_{k+1}-t_{k} \\
X_{j}=X\left(t_{j}\right), w_{k}=w_{t_{k}}
\end{gathered}
$$

We replace $w_{k} \Delta t_{k}$ in (3) by $\Delta V_{k}$ such that $E\left(V_{t}\right)_{t \geq 0}$ is stochastic process .let $V_{t}=B_{t}$ $3 \rightarrow X_{k}=X_{0}=\sum_{j=0}^{k-1} b\left(t_{j}, X_{j}\right) \Delta t_{j}+\sum_{j=0}^{k-1} \sigma\left(t_{j}, X_{j}\right) \Delta B_{j}$

Then apply the usual integration notation we should obtain

$X_{t}=X_{0}=\int_{0}^{t} b\left(s, X_{s}\right) d s+\int_{0}^{t} \sigma\left(s, X_{s}\right) d B_{s}$ 
The last formula (5) is the ito integral.

\section{The other definition of ito integral:}

For function $f \in V, V=V(s, t)$ be the class of functions $f(t, w):[0, \infty[\times \Omega \rightarrow \mathbb{R}$ we define the ito integral $I(f)(w)=\int_{s}^{T} f(t, w) d B_{t}(w)(6)$

Lemma1-2 :( the ito isometry):

If $\emptyset(t, w)$ is bounded and elementary (if $\emptyset \in V$ is called elementary if it has the form $\left.\emptyset(t, w)=\sum_{j} e_{j}(w) \cdot X_{\left[t_{j}, t_{j+1}\right](t)}\right)$

Then $E\left[\left(\int_{S}^{T} \emptyset(t, w) d B_{t}(w)\right)^{2}\right]=E\left[\int_{S}^{T} \emptyset(t, w)^{2} d t\right]$

Proof:

See page 26 in[1]

\section{Definition (ito process):}

The 1-dimention ito process:

Let $B_{t}$ be 1- dimensional Brownian motion on $(\boldsymbol{\Omega}, \mathcal{F}, \boldsymbol{P})$ An ito processes is stochastic process $X_{t}$ in of the form $X_{t}=X_{0}+\int_{0}^{t} u(S, w) d S+\int_{0}^{t} v(S, w) d B_{S}$

Where $v \in w_{H}$, so that

\section{Example1-2:}

$$
P\left[\int_{0}^{t} v(S, w)^{2} d S<\infty \quad \forall t \geq 0\right]=1
$$

Prove directly from the definition of ito integrals that:

$\int_{0}^{t} S d B_{s}=t B_{t}-\int_{0}^{t} B_{s} d s$

\section{Solution:}

Using the notation $\Delta x_{j}=x_{j+1}-x_{j}$

There fore

$$
\sum_{j} \Delta\left(S_{j} B_{j}\right)=\sum_{j} S_{j} \Delta B_{j}+\sum_{j} B_{j+1} \Delta S_{j}
$$

By integrating

$$
\sum_{j} \Delta\left(t_{j} B_{j}\right)=\sum_{j} t_{j} \Delta B_{j}+\sum_{j} B_{j+1} \Delta t_{j}
$$

\section{Example2-2:}

$$
\begin{gathered}
\int_{0}^{t} \Delta\left(t_{j} B_{j}\right)=\int_{0}^{t} S d B_{S}+\int_{0}^{t} B_{S} d s \\
t B_{t}=\int_{0}^{t} S d B_{S}+\int_{0}^{t} B_{S} d S \\
\int_{0}^{t} S d B_{S}=t B_{t}-\int_{0}^{t} B_{S} d S
\end{gathered}
$$

Prove from the definition of ito integral that

\section{Solution:}

$$
\int_{0}^{t} B_{S}^{2} d B_{S}=\frac{1}{3} B_{t}^{3}-\int_{0}^{t} B_{S} d S
$$

Where $\sum_{j}\left(\Delta B_{j}\right)^{2} \rightarrow B_{j}$

$$
\begin{gathered}
B_{t}^{3}=\sum_{j} \Delta B_{j}^{3}=\sum_{j} B_{j+1}^{3}-B_{j}^{3} \\
=\sum_{j}\left(B_{j+1}-B_{j}\right)^{3}+3 B_{j+1}^{2} B_{j}-3 B_{j}^{2} B_{j+1} \\
=\sum_{j}\left(B_{j+1}-B_{j}\right)^{3}+3 \sum_{j} B_{j+1} B_{j}\left(B_{j+1}-B_{j}\right) \\
B_{t}^{3}=\sum_{j}\left(\Delta B_{j}\right)^{3}+3 \sum_{j} B_{j+1} B_{j} \Delta B_{j} \\
\sum_{j} B_{j} B_{j} \Delta B_{j}=\frac{1}{3} B_{t}^{3}-\frac{1}{3} \sum_{j}\left(\Delta B_{j}\right)^{3} \\
\sum_{j} B_{j} B_{j} \Delta B_{j}=\frac{1}{3} B_{t}^{3}-\sum_{j}\left(\Delta B_{j}\right)^{2} d t_{j}
\end{gathered}
$$

There fore $\quad \sum_{j} B_{j} B_{j} \Delta B_{j}=\frac{1}{3} B_{t}^{3}-\sum_{j} B_{j} d t_{j}$

By integrating the last equation from 0 into $t$ we get 


$$
\int_{0}^{t} B_{S}^{2} d B_{S}=\frac{1}{3} B_{t}^{3}-\int_{0}^{t} B_{S} d S
$$

(3)ito formula:

\section{Theorem1-3:}

The 1-dimensional ito formula

Let $X_{t}$ be an ito process given by

$$
\text { Let } g(t, x) \in C^{2}([0, \infty[\times R) \text {, then }
$$

$$
d X_{t}=u d t+v d B_{t}
$$

$Y_{t}=g\left(t, X_{t}\right)$ is also an ito process and

$d Y_{t}=\frac{\partial g}{\partial t}\left(t, X_{t}\right) d t+\frac{\partial g}{\partial X}\left(t, X_{t}\right) d X_{t}+\frac{1}{2 \partial} \frac{\partial^{2} g}{X^{2}}\left(t, X_{t}\right)\left(d X_{t}\right)^{2}$

Where $\left(d X_{t}\right)^{2}=d X_{t} \times d X_{t}$

$$
\begin{gathered}
d t \times d t=d t d B_{t}=d B_{t} \times d t=0 \\
d B_{t} \times d B_{t}=d t
\end{gathered}
$$

Proof:

See page 44in[1]

Example1-3:

Use ito formula to write the stochastic process

On the standard form

$$
Y_{t}=B_{t}^{2}
$$

\section{Solution:}

$$
d Y_{t}=u(t, w) d t+v(t, w) d B_{t}
$$

By the 1-dimentional ito formula:

Since $\quad Y_{t}=B_{t}^{2}$

There fore $d\left(B_{t}^{2}\right)=2 B_{t} d B_{t}+d t$

$d\left(B_{t}^{2}\right)=d t+2 B_{t} d B_{t}$

Where $u(t, w)=1, \quad v(t, w)=2 B_{t}$

\section{Example2-3:}

Use the ito formula to write the stochastic process

\section{Solution:}

$$
Y_{t}=\left(2+t+e^{B_{t}}\right)
$$

By the 1-dimensional ito formula:

$$
\begin{gathered}
d Y_{t}=\frac{\partial g}{\partial t} d t+\frac{\partial g}{\partial X} d B_{t}+\frac{1}{2} \frac{\partial^{2} g}{\partial X^{2}}\left(d B_{t}\right)^{2} \\
d Y_{t}=d t+e^{B_{t}} d B_{t}+\frac{1}{2} e^{B_{t}} d t \\
d Y_{t}=\left(1+\frac{1}{2} e^{B_{t}}\right) d t+e^{B_{t}} d B_{t}
\end{gathered}
$$

Where $u(t, w)=\left(1+\frac{1}{2} e^{B_{t}}\right), v(t, w)=e^{B_{t}}$

\section{The multi-dimensional ito formula:}

Let $B_{1}(t, w)=\left(B_{1}(t, w), \ldots, B_{m}(t, w)\right)$

Denotes m-dimensional Brownian motion.

If each of the processes $u_{i}(t, w), v_{i j}(t, w)$ satisfies equation (9) then we can form the following n-ito processes $\left\{\begin{array}{c}d X_{1}=u_{1} d t+v_{11} d B_{1}+\cdots+v_{1 m} d B_{m} \\ \vdots \\ d X_{n}=u_{n} d t+v_{n 1} d B_{1}+\cdots+v_{n m} d B_{m}\end{array}\right.$

Or in matrix notation simply

$d X(t)=u d t+v d B_{t}$
Where $X(t)=\left[\begin{array}{c}X_{1}(t) \\ \vdots \\ X_{n}(t)\end{array}\right], u=\left[\begin{array}{c}u_{1} \\ \vdots \\ u_{n}\end{array}\right], v=\left[\begin{array}{ccc}v_{11} & \cdots & v_{1 m} \\ \vdots & \ddots & \vdots \\ v_{n 1} & \cdots & v_{n m}\end{array}\right], d B_{t}=\left[\begin{array}{c}d B_{1} \\ \vdots \\ d B_{2}\end{array}\right]$

Theorem2-3 (the general ito formula):

Let $d X(t)=u d t+v d B_{t}$

Let $g(t, X)=\left(g_{1}(t, X), g_{2}(t, X), \cdots, g_{p}(t, X)\right)$

Be $C^{2}:\left[0, \infty\left[\times \mathbb{R}^{n} \rightarrow \mathbb{R}^{p}\right.\right.$

Then $Y(t, w)=g(t, X(t))$ 
The general ito formula is

Where $d B_{i} d B_{j}=\delta_{i j} d t, d B_{i} d t=d t d B_{i}=0$

$$
d Y_{k}=\frac{\partial g_{k}}{\partial t}(t, X) d t+\sum_{i} \frac{\partial g_{k}}{\partial X_{i}}(t, X) d X_{i}+\frac{1}{2} \sum_{i, j} \frac{\partial^{2} g_{k}}{\partial X_{i} \partial X_{j}}(t, X) d X_{i} d X_{j}
$$

\section{Proof:}

Seepage49in[1]

\section{Example3-3:}

Use the general ito formula to write the stochastic process $Y_{t}$ on the standard form $d Y_{t}=u(t, w) d t+v(t, w) d B_{t}$ If $Y_{t}=\left(B_{1}(t)+B_{2}(t)+B_{3}(t), B_{2}^{2}(t)-B_{1}(t)-B_{3}(t)\right)$

\section{Solution:}

Or in matrix form

$$
\begin{aligned}
d X_{1}(t) & =\frac{\partial g}{\partial t} d t+\frac{\partial g}{\partial X_{t}} d B_{1}(t)+\frac{\partial g}{\partial X_{t}} d B_{2}(t)+\frac{\partial g}{\partial X_{t}} d B_{3}(t) \\
=d B_{1}(t)+ & d B_{2}(t)+d B_{3}(t) \\
d X_{2}(t) & =\frac{\partial g}{\partial t} d t+\frac{\partial g}{\partial X_{t}} d B_{t}(t)+\frac{1}{2} \frac{\partial^{2} g}{\partial X_{t}{ }^{2}}\left(d B_{t}\right)^{2}+\frac{\partial g}{\partial X_{t}} d B_{3}(t) \\
= & 2 B_{2} d B_{2}+\frac{1}{2} \cdot 2 d t-B_{3} d B_{1}(t)-B_{1} d B_{3}(t)
\end{aligned}
$$

$$
d X_{t}=\left[\begin{array}{l}
d X_{1} \\
d X_{2}
\end{array}\right]=\left[\begin{array}{l}
0 \\
1
\end{array}\right] d t+\left[\begin{array}{ccc}
1 & 1 & 1 \\
-B_{3}(t) & 2 B_{2}(t) & -B_{1}(t)
\end{array}\right]\left[\begin{array}{l}
d B_{1}(t) \\
d B_{2}(t) \\
d B_{3}(t)
\end{array}\right]
$$

\section{4-Solution of stochastic differential equation:}

The solution of stochastic differential equations

$\frac{d X_{t}}{d t}=b\left(t, X_{t}\right)+\sigma\left(t, X_{t}\right) W_{t}, b\left(t, X_{t}\right), \sigma\left(t, X_{t}\right) \in \mathbb{R}$

$W_{t}$ Is the 1-dimentional white noise

Is $X_{t}=X_{0}+\int_{0}^{t} b\left(s, X_{s}\right) d s+\int_{0}^{t} \sigma\left(s, X_{s}\right) d B_{s}$

Or in differential form

\section{Example1-4:}

$$
d X_{t}=b\left(t, X_{t}\right) d t+\sigma\left(t, X_{t}\right) d B_{t}
$$

Verify that the given processes solve corresponding stochastic differential equation: $X_{t}=e^{B_{t}}$ Solves $d X_{t}=$ $\frac{1}{2} X_{t} d t+X_{t} d B_{t}$

\section{Solution:}

\section{Let $X_{t}=g$}

Use the 1-dimentional ito formula

We get, $\quad d X_{t}=e^{B_{t}} d B_{t}+\frac{1}{2} e^{B_{t}}\left(d B_{t}\right)^{2}$

$$
d Y_{t}=\frac{\partial g}{\partial t} d t+\frac{\partial g}{\partial X} d B_{t}+\frac{1}{2} \frac{\partial^{2} g}{\partial X^{2}}\left(d B_{t}\right)^{2}
$$

$$
\begin{aligned}
& \Rightarrow d X_{t}=X_{t} d B_{t}+\frac{1}{2} X_{t} d t \\
& \Rightarrow d X_{t}=\frac{1}{2} X_{t} d t+X_{t} d B_{t}
\end{aligned}
$$

\section{Example 2-4:}

Verify that the given processes solve corresponding stochastic differential equation

$\left(X_{1}, X_{2}\right)=\left(\cosh \left(B_{t}\right), \sinh \left(B_{t}\right)\right)$

Solves $\left[\begin{array}{l}d X_{1} \\ d X_{2}\end{array}\right]=\frac{1}{2}\left[\begin{array}{l}X_{1} \\ X_{2}\end{array}\right] d t+\left[\begin{array}{l}X_{2} \\ X_{1}\end{array}\right] d B_{t}$

\section{Solution:}

We use the general ito formula

$$
\begin{gathered}
d Y_{k}=\frac{\partial g_{k}}{\partial t}(t, X) d t+\sum_{i} \frac{\partial g_{k}}{\partial X_{i}}(t, X) d X_{i}+\frac{1}{2} \sum_{i, j} \frac{\partial^{2} g_{k}}{\partial X_{i} \partial X_{j}}(t, X) d X_{i} d X_{j} \\
d X_{1}=\frac{\partial g}{\partial t} d t+\frac{\partial g}{\partial X} d X_{t}+\frac{1}{2} \frac{\partial^{2} g}{\partial X^{2}}\left(d X_{t}\right)^{2}
\end{gathered}
$$


$=\sinh \left(B_{t}\right) d B_{t}+\frac{1}{2} \cosh \left(B_{t}\right)\left(d B_{t}\right)^{2}=$

$$
\begin{gathered}
=X_{2} d B_{t}+\frac{1}{2} X_{1} d t \\
=\frac{1}{2} X_{1} d t+X_{2} d B_{t} \\
d X_{2}=\frac{\partial g}{\partial t} d t+\frac{\partial g}{\partial X} d X_{t}+\frac{1}{2} \frac{\partial^{2} g}{\partial X^{2}}\left(d X_{t}\right)^{2}=\cosh B_{t} d B_{t}+\frac{1}{2} \sinh B_{t}\left(d B_{t}\right)^{2} \\
=\frac{1}{2} X_{2} d t+X_{1} d B_{t}
\end{gathered}
$$

Therefore, the given processes solves the given stochastic differential equation $\mathrm{s}$

\section{Example3- 4:}

\section{(exponential growth with noise)}

Solve the following stochastic differential equation

$d X_{t}=X_{t} d t+d B_{t}$

\section{Solution:}

Multiply both sides with the integrating factor $e^{-t}$

Thus,

On the other hand applying the stochastic rule

$$
e^{-t} d X_{t}=e^{-t} X_{t} d t+e^{-t} d B_{t}
$$

We have

$$
d\left(X_{t} Y_{t}\right)=X_{t} d Y_{t}+Y_{t} d X_{t}+d X_{t} d Y_{t}
$$

$$
\begin{aligned}
& d\left(e^{-t} X_{t}\right)=X_{t} d e^{t}+e^{-t} d X_{t}+d e^{-t} d X_{t} \\
& =-e^{-t} X_{t} d t+e^{-t} d X_{t}-e^{-t}\left(X_{t} d t+d B_{t}\right) \\
& =-e^{-t} X_{t} d t+e^{-t}\left(X_{t} d t+d B_{t}\right) X_{t}-e^{-t} d t\left(X_{t} d t+d B_{t}\right) \\
& =e^{-t} d B_{t} \\
& =-e^{-t} X_{t} d t+e^{-t} X_{t} d t+e^{-t} d B_{t}-e^{-t} X_{t}(d t)^{2}+e^{-t} d t d B_{t}
\end{aligned}
$$

Integrating both sides, we have

We have the expected value

$$
\begin{aligned}
& \int_{0}^{t} d\left(e^{-t} X_{t}\right)=\int_{0}^{t} e^{-t} d B_{t} \\
& e^{-t} X_{t}-X_{0}=\int_{0}^{t} e^{-t} d B_{t}, X_{0}=C \\
& e^{-t X_{t}}=C+\int_{0}^{t} e^{-t} d B_{t} \\
& X_{t}=C e^{t}+e^{t} \int_{0}^{t} e^{-t} d B_{t}
\end{aligned}
$$

$$
\begin{aligned}
& E\left[X_{t}\right]=E\left[C e^{t}\right]+E\left[e^{t} \int_{0}^{t} e^{-t} d B_{t}\right] \\
& =C e^{t}
\end{aligned}
$$

\section{Conclusion:}

The above method of solution of some stochastic differential equations is a good method for the equations which contain the random variable and their solution depends on the given an ito integral and an ito formula which shows above .in the next papers I will discuss the solution of second order stochastic differential equation also discuss the solution of partial stochastic differential equations.

\section{References:}

[1] Bernt Qksendal (2006) :Stochastic differential equations an introduction with application, sixth edition Springer -Verlag Berlin Heidelberg New York

[2] Bernt Qksendal (1998): Stochastic differential equations an introduction with application, fifth edition Springer -Verlage Berlin Heidelberg Italy

[3] John Kerl (2008): Problems in stochastic differential equations, Springer

[4] Protter, p.(1990):stochastic integration and differential equations,Springer -Verlag

[5] Gihman, 1.1., Skorohod, A.V.(1974a):stochastic differential equations, Springer -Veralg

[6] Hui- Hsiung Kuo (2006): introduction to stochastic integration -Springer

[7] William, D.(1981)(edior):stochastic integrals -lecture notes in mathematics, vol.851-Springer -Verlag

[8] Karatzas, I., Shereve, S.E. (1991): Brownian motion and stochastic calculus. Second edition.Springer-Verlag 